\title{
ARITHMETIC OF ORDINALS WITH APPLICATIONS TO THE THEORY OF ORDERED ABELIAN GROUPS
}

\author{
PHILIP W. CARRUTH
}

1. Introduction. ${ }^{1}$ The operations of addition and multiplication of ordinals do not behave as well as one might desire. For example, the commutative laws are not valid, and the distributive law is valid on only one side. Consequently, we make definitions of a sum and product that do not have such defects.

A binary operation, $\alpha \oplus \beta$, on ordinals is termed a natural sum if $\alpha \oplus \beta$ is a well-determined ordinal for any two ordinals, $\alpha$ and $\beta$, such that:

(1) $\alpha \oplus \beta=\beta \oplus \alpha$,

(2) $(\alpha \oplus \beta) \oplus \delta=\alpha \oplus(\beta \oplus \delta)$,

(3) $\alpha \oplus 0=\alpha$,

(4) $\delta \oplus \alpha>\delta \oplus \beta$ if and only if $\alpha>\beta$,

where $\delta$ is any ordinal.

Throughout this paper, $\sigma(\alpha, \beta)$ will denote the natural sum defined by Hessenberg. ${ }^{2}$ It is the unique natural sum satisfying the condition that $\omega^{\alpha} \cdot m+\omega^{\beta} \cdot n=\sigma\left(\omega^{\alpha} m, \omega^{\beta} n\right)$, where $\alpha$ and $\beta$ are any two ordinals such that $\alpha \geqq \beta$, and where $m$ and $n$ are any two positive integers. $\sigma(\alpha, \beta)$ shall be shown to be the "smallest" natural sum, and it shall be shown to be the best bound for the order type of the join of two well-ordered subsets, of respective order types $\alpha$ and $\beta$, of an ordered set.

A binary operation, $\alpha \otimes \beta$, on ordinals is termed a natural product if $\alpha \otimes \beta$ is a well-determined ordinal for any two ordinals, $\alpha$ and $\beta$, such that:

(1) $\alpha \otimes \beta=\beta \otimes \alpha$,

(2) $(\alpha \otimes \beta) \otimes \delta=\alpha \otimes(\beta \otimes \delta)$,

(3) $\alpha \otimes 1=\alpha$,

(4) $\alpha \otimes \delta>\beta \otimes \delta$ if and only if $\alpha>\beta$,

(5) $\sigma(\alpha \otimes \beta, \alpha \otimes \delta)=\alpha \otimes \sigma(\beta, \delta)$,

(6) $\omega^{\alpha} \otimes \omega^{\beta}=\omega^{\gamma}$,

where $\delta$ is any ordinal, and where $\gamma=\gamma(\alpha, \beta)$ is a suitable ordinal.

Presented to the Society, September 5, 1941; received by the editors June 6, 1941. The writer is indebted to Professor Reinhold Baer for his advice in the preparation of this paper.

1 The writer presupposes familiarity with the material on ordinals found in F. Hausdorff's Mengenlehre.

${ }^{2}$ G. Hessenberg, Grundbegriffe der Mengenlehre, Abhandlungen der Fries'schen Schule, (n. s.), 1.4, Göttingen, 1906, no. 75. 
It may be noted that the ordinal $\gamma$ above is a natural sum of $\alpha$ and $\beta$. If we impose the condition that this ordinal be equal to $\sigma(\alpha, \beta)$, we obtain the unique natural product defined by Hausdorff. ${ }^{3}$ This particular natural product will be denoted by $\pi(\alpha, \beta)$ throughout this paper. We shall show that $\pi(\alpha, \beta)$ is the "smallest" natural product and that it is the best bound for the order type of a certain rectangular array of ordered elements that has " $\alpha$ rows" and " $\beta$ columns." Another application of Hausdorff's natural product will be given in determining a bound for the order type of the semi-group generated by a well-ordered set of positive elements in an ordered Abelian group. ${ }^{4}$

It is known that every ordinal $\alpha$ may be represented in the form, $\alpha=\sum_{i=1}^{n} \omega^{\alpha} a_{i}$, where $\omega>n, a_{1}, a_{2}, \cdots, a_{n} \geqq 0$ and $\alpha \geqq \alpha_{1}>\alpha_{2}>\cdots$ $>\alpha_{n} \geqq 0$. Henceforth in this paper, when an ordinal is written in summation form, it will be assumed that the summation satisfies the above requirements.

2. Natural sums and products of ordinals. We first prove the following theorem.

THEOREM 1. Let $\tau \oplus \rho$ be any natural sum.

I. Let $T$ and $R$ be well-ordered subsets, of respective order types $\tau$ and $\rho$, of an ordered set of elements. Then the set of elements in the join of $R$ and $T$, that is, $R+T$, is well-ordered and has order type less than or equal to $\tau \oplus \rho$.

II. There exists a well-ordered subset $R$, of order type $\rho$, of an ordered set, and a well-ordered subset $T$, of order type $\tau$, of the same ordered set, such that the order type of $R+T$ is exactly $\sigma(\rho, \tau)$.

III. $\sigma(\rho, \tau) \leqq \tau \oplus \rho$.

IV. $\tau+\rho \leqq \tau \oplus \rho .^{5}$

Proof of I. I is true for $\tau=0$ and for all $\rho$. Assume it to be true for all $\rho$ and for all $\tau$ less than $\alpha$. It is true for $\rho=0$ and $\tau=\alpha$. Assume it to be true for all $\rho$ less than $\beta$ and $\tau=\alpha$. We shall now prove I to be true for $\rho=\beta$ and $\tau=\alpha$.

It is easily verified that $R+T$ is well-ordered for $\rho=\beta$ and $\tau=\alpha$. Suppose the order type of $R+T$ is greater than $\beta \oplus \alpha$. Then $R+T$ has a segment $Z$ of order type $\beta \oplus \alpha$. By our induction hypothesis, it is

${ }^{3}$ F. Hausdorff, Mengenlehre, 3rd edition, p. 70.

${ }^{4}$ For a definition of an ordered Abelian group, see S. MacLane, The uniqueness of the power series representation of certain fields with valuations, Annals of Mathematics, (2), vol. 39 (1938), p. 371.

${ }^{5} \sigma(\tau, \rho)$ may be larger than the maximum $\{\tau+\rho, \rho+\tau\}$, for example, let $\tau=\rho=\omega+1$. Then $\tau+\rho=\rho+\tau=\omega 2+1$, and $\sigma(\rho, \tau)=\omega 2+2$. 
seen that the order type of the subset of elements of $T$ that are in $Z$ must be $\alpha$, since $\alpha \oplus \beta>\mu \oplus \beta$ for $\alpha>\mu$. Likewise the order type of the subset of elements of $R$ that are in $Z$ must be $\beta$. The elements of $R$ that are in $Z$ form a segment of $R$, and the elements of $T$ that are in $Z$ form a segment of $T$. Hence the set $R+T$ is the same as the segment $Z$ of $R+T$. This gives us a contradiction, proving $\mathrm{I}$.

ProOF OF II. Let $\rho=\sum_{i=1}^{n} \omega^{\alpha_{2}} a_{i}$, and $\tau=\sum_{i=1}^{n} \omega^{\alpha} b_{i}$. Then $\sigma(\rho, \tau)$ is equal to $\sum_{i=1}^{n} \omega^{\alpha}\left(a_{i}+b_{i}\right)$. Let $R$ be a set of elements of order type $\rho$. Then $R$ is the join of sets $R_{i}, 1 \leqq i \leqq n$, where $R_{i}$ has order type $\omega^{\alpha i} a_{i}$, and where each element in $R_{i}$ is smaller than every element in $R_{i+1}$.

Let $T$ be a set of elements of order type $\tau$. Then $T$ is the join of sets $T_{i}, 1 \leqq i \leqq n$, where $T_{i}$ has order type $\omega^{\alpha} b_{i}$, and where each element in $T_{i}$ is smaller than every element in $T_{i+1}$.

By letting each element in $T_{i}$ be greater than every element in $R_{i}$ and smaller than every element in $R_{i+1}$, we form a set $R+T$ of order type $\sigma(\rho, \tau)$.

III is an immediate consequence of I and II, and IV is an immediate consequence of $I$.

An ordered set of elements, $A=\left\{a_{\alpha \beta}\right\}, 0 \leqq \alpha<\tau, 0 \leqq \beta<\mu$, where $\tau$ and $\mu$ are any two ordinals, is an ordered $\tau, \mu$-block if $a_{\alpha \beta}<a_{\alpha \gamma}, a_{\beta \alpha}<a_{\gamma \alpha}$ for $\beta<\gamma$. An ordered $\tau, \mu$-block $A$ is said to be less than an ordered $\rho$, $\lambda$-block $B$, that is, $A<B$, if each element in $A$ is considered to be less than every element in $B$. Two ordered $\tau, \mu$-blocks, $A=\left\{a_{\alpha \beta}\right\}$ and $B=\left\{b_{\alpha \beta}\right\}$, are congruent if the following two conditions are equivalent: (1) $a_{\alpha \beta}<a_{\rho \lambda}$, (2) $b_{\alpha \beta}<b_{\rho \lambda}$.

THEOREM 2. Let $\tau \otimes \mu$ be any natural product.

I. Let $A=\left\{a_{\alpha \beta}\right\}$ be an ordered $\tau, \mu$-block. Then $A$ is well-ordered and has order type less than or equal to $\tau \otimes \mu$.

II. There exists an ordered $\tau, \mu$-block of order type exactly $\pi(\tau, \mu)$.

III. $\pi(\tau, \mu) \leqq \tau \otimes \mu$.

IV. $\tau \mu \leqq \tau \otimes \mu .^{6}$

Proof of I. Suppose that $A$ is not well-ordered. Then there exists a subset $B$ of $A, a_{\alpha_{1} \beta_{1}}>a_{\alpha_{2} \beta_{2}}>\cdots$, of order type $\omega^{*}$. Since the $\alpha_{i}$ are well-ordered, we may choose a chain $C, a_{\delta_{1} \gamma_{1}}>a_{\delta_{2} \gamma_{2}}>\cdots$, of order type $\omega^{*}$, from the set $B$ such that $\delta_{1} \leqq \delta_{2} \leqq \cdots$. Since the $\gamma_{i}$ are wellordered, there exists a $\gamma_{i}$, say $\gamma_{k}$, such that $\gamma_{k} \geqq \gamma_{1}$. But then $a_{\delta_{k} \gamma_{k}} \geqq a_{\delta_{1} \gamma_{1}}$, which is a contradiction.

The set $A$ has order type less than or equal to $\tau \otimes \mu$ for $\tau=1$ and all $\mu$. Assume this to be true for all $\mu$ and for all $\tau$ less than $\delta$. It is

${ }^{6} \pi(\rho, \tau)$ may be larger than the maximum $\{\tau \rho, \rho \tau\}$, for example, let $\tau=\rho=\omega+1$. Then $\tau \rho=\rho \tau=\omega^{2}+\omega+1$, and $\pi(\rho, \tau)=\omega^{2}+\omega 2+1$. 
true for $\mu=1$ and $\tau=\delta$. Assume it to be true for all $\mu$ less than $\gamma$ and $\tau=\delta$. We shall now prove $A$ has order type less than or equal to $\tau \otimes \mu$ for $\mu=\gamma$ and $\tau=\delta$.

Let $\delta=\omega^{\alpha_{1}} m_{1}+\cdots+\omega^{\alpha_{r}} m_{r}$ and $\gamma=\omega^{\beta_{1}} n_{1}+\cdots+\omega^{\beta_{s}} n_{\mathbf{s}}$.

Case I. $m_{1}>1$ or $r>1$.

Let $\rho=\omega^{\alpha_{1}} m_{1}+\cdots+\omega^{\alpha_{r}}\left(m_{r}-1\right)$, where $m_{r}-1 \geqq 0$. By hypothesis, $\delta>\rho>0$ and $\delta>\omega^{\alpha r}$.

Let $T_{1}$ be the set $\left\{a_{\alpha \beta}\right\}$, where $0 \leqq \alpha<\rho$ and $0 \leqq \beta<\gamma$. Let $T_{2}$ be the set $\left\{a_{\alpha \beta}\right\}$, where $\rho \leqq \alpha<\delta$ and $0 \leqq \beta<\gamma$. Then $A$ is the set $T_{1}+T_{2}$. By our induction hypotheses, the order type of $T_{1}$ is less than or equal to $\rho \otimes \gamma$. Likewise, the order type of $T_{2}$ is less than or equal to $\omega^{\alpha_{r}} \otimes \gamma$. Theorem 1 implies that the order type of $A$ is less than or equal to $\sigma\left(\rho \otimes \gamma, \omega^{\alpha r} \otimes \gamma\right)=\gamma \otimes \sigma\left(\rho, \omega^{\alpha r}\right)=\gamma \otimes \delta$.

Because of the symmetry, $A$ has order type less than or equal to $\tau \otimes \mu$ for $\mu=\gamma$ and $\tau=\delta$, if $n_{1}>1$ or $s>1$.

Case II. $m_{1}=r=n_{1}=s=1$, that is, $\delta=\omega^{\alpha_{1}}$ and $\gamma=\omega^{\beta_{1}}$.

Suppose that the order type of $A$ is greater than $\delta \otimes \gamma$. Then $A$ has a segment $Z$ of order type $\delta \otimes \gamma$. Let $A=Z+Y$. Let $a_{\lambda \epsilon}$ be the smallest element in $Y$. Then $\delta>\lambda$ and $\gamma>\epsilon$.

Let $T_{1}$ be the set $\left\{a_{\alpha \beta}\right\}$, where $0 \leqq \alpha<\delta$ and $0 \leqq \beta<\epsilon$. Let $T_{2}$ be the set $\left\{a_{\alpha \beta}\right\}$, where $0 \leqq \alpha<\lambda$ and where $\epsilon \leqq \beta<\gamma$. Let $W=T_{1}+T_{2}$.

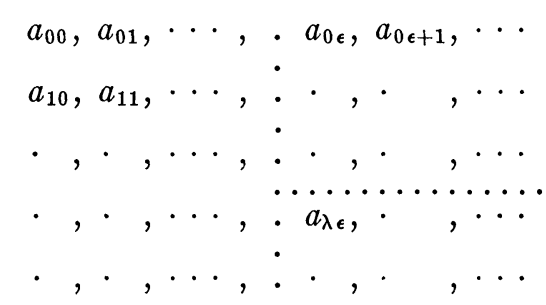

The set at the left of the dotted line is $T_{1}$, and that in the upper righthand corner is $T_{2}$.

$W$ certainly contains $Z$. Therefore, according to our supposition, the order type of $W$ is greater than or equal to $\delta \otimes \gamma$.

By our induction hypothesis, the order type of $T_{1}$ is less than or equal to $\delta \otimes \epsilon$, and the order type of $T_{2}$ is less than or equal to $\gamma \otimes \lambda$. Theorem 1 implies that the order type of $W$ is less than or equal to $\sigma(\delta \otimes \epsilon, \gamma \otimes \lambda)$. According to (6) of the definition of a natural product, $\delta \otimes \gamma=\omega^{\eta}$, where $\eta$ is some ordinal. Likewise, by (4) of this definition, it is seen that $\delta \otimes \epsilon<\omega^{\eta}$ and that $\gamma \otimes \lambda<\omega^{\eta}$. The definition of $\sigma(\alpha, \beta)$ implies that $\sigma(\delta \otimes \epsilon, \gamma \otimes \lambda)<\omega^{\eta}=\delta \otimes \gamma$. This then is a contradiction which completes the proof of I.

ProOF OF II. Let $\tau=\omega^{\alpha} a_{1}+\cdots+\omega^{\alpha r} a_{r}$ and $\mu=\omega^{\beta_{1}} b_{1}+\cdots+\omega^{\beta s} b_{s}$. 
Then $\pi(\tau, \mu)=\sum_{i, j} \omega^{\sigma\left(\alpha_{i}, \beta_{j}\right)} a_{i} b_{j}=\sum_{k} \omega^{\gamma_{k}} c_{k}$ where $c_{k}=\sum{ }^{k} a_{i} b_{j}$, where $\sum^{k}$ is over the finite number of pairs such that $\sigma\left(\alpha_{i}, \beta_{j}\right)=\gamma_{k}$.

Any ordered $\tau, \mu$-block, $A=\left\{a_{\alpha \beta}\right\}$, may be broken up into ordered $\omega^{\alpha_{i}} a_{i}, \omega^{\beta_{i}} b_{j}$-blocks, $D(i, j)=\left\{a_{\alpha \beta}\right\}$, where $\omega^{\alpha_{1}} a_{1}+\cdots+\omega^{\alpha_{i-1}} a_{i-1} \leqq \alpha$ $<\omega^{\alpha_{1}} a_{1}+\cdots+\omega^{\alpha} a_{i}$, and $\omega^{\beta_{1}} b_{1}+\cdots+\omega^{\beta_{j-1}} b_{j-1} \leqq \beta<\omega^{\beta_{1}} b_{1}+\cdots$ $+\omega^{\beta} b_{j}$.

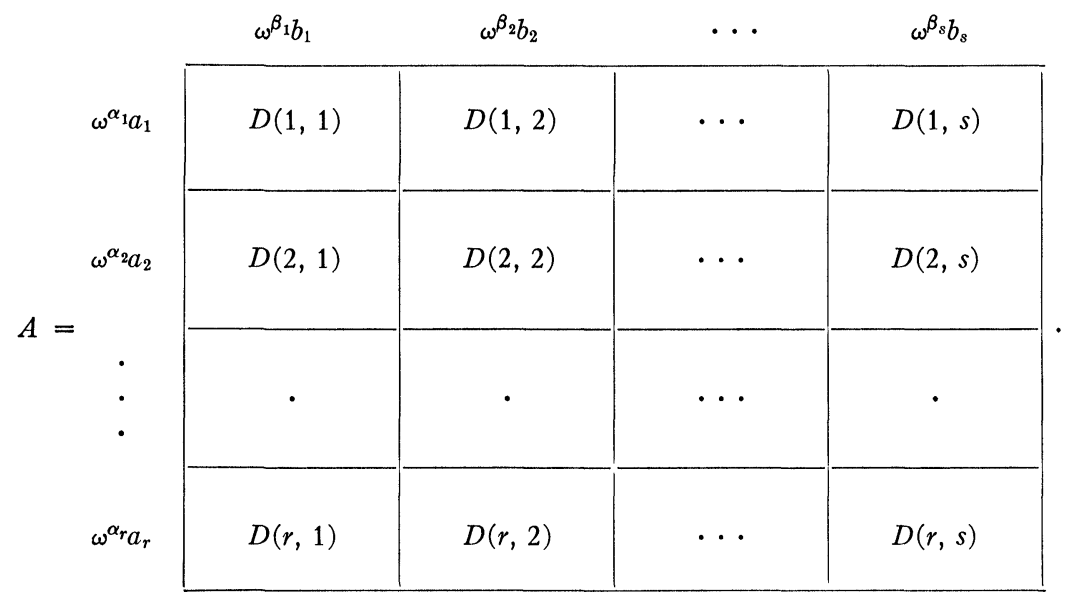

Suppose that we could order our blocks $D(i, j)$ in such a way that each $D(i, j)$ would have order type exactly $\pi\left(\omega^{\alpha i} a_{i}, \omega^{\beta_{j}} b_{j}\right)=\omega^{\sigma\left(\alpha_{i}, \beta_{j}\right)} a_{i} b_{j}$. By then letting $D(i, j)$ be less than $D(k, m)$ if $\sigma\left(\alpha_{i}, \beta_{j}\right)>\sigma\left(\alpha_{k}, \beta_{m}\right)$ or if $\sigma\left(\alpha_{i}, \beta_{j}\right)=\sigma\left(\alpha_{k}, \beta_{m}\right)$ and $i>k$, we would have an ordered $\tau, \mu$-block of order type exactly $\pi(\tau, \mu)$. Thus it is seen that it suffices to prove II for the special case that $\tau=\omega^{\alpha} a$ and $\mu=\omega^{\beta} b$.

Hence we now assume that $\tau=\omega^{\alpha} a$ and that $\mu=\omega^{\beta} b$. Let $\alpha=\omega^{\gamma_{1}} f_{1}+\cdots+\omega^{\gamma} f_{t}$ and $\beta=\omega^{\delta_{1}} d_{1}+\cdots+\omega^{\delta v} d_{v}$. We first assume that $t=0$. In this case $\alpha=0$ and $\tau=a$. If we let $a_{\lambda_{\eta}}<a_{\theta_{\rho}}$ for $\lambda<\theta$, it is seen that an ordered $\tau, \mu$-block is formed of order type $\mu \tau=\pi(\mu, \tau)$. Assume that II is true for all integers $t$ less than $k$ and for all integers $v$. We shall now prove II to be true when $t=k$.

$$
\omega^{\alpha}=\omega^{\omega^{\gamma} f_{1}+\cdots+\omega^{\gamma} \gamma_{k-1} f_{k-1} \cdot \omega^{\omega} \gamma_{k f k}} .
$$

Let

$$
\phi=\omega^{\omega \gamma_{1} f_{1}+\cdots+\omega \gamma_{k-1} f_{k-1}}
$$

Then

$$
\begin{aligned}
& \omega^{\alpha}=\phi \cdot \omega^{\omega^{\gamma} k f_{k}}=\pi\left(\phi, \omega^{\omega^{\alpha} k f_{k}}\right), \\
& \omega^{\beta}=\omega^{\omega^{\delta} d_{1}+\cdots+\omega^{\delta} d_{e}} \cdot \omega^{\omega^{\delta_{e+1}} d_{e+1}+\cdots+\omega^{\delta} d_{v}}
\end{aligned}
$$


where $\delta_{\theta} \geqq \gamma_{k}>\delta_{\theta+1}, 0 \leqq e \leqq v$. Let

$$
\lambda=\omega^{\omega^{\delta}{ }_{1}+\cdots+\omega^{\delta} e_{e}}, \quad \eta=\omega^{\omega^{\delta} e+1} d_{e+1}+\cdots+\omega^{\delta} v_{d v} .
$$

If $e=0$, let $\lambda=1$. If $e=v$, let $\eta=1$.

$$
\begin{aligned}
\pi\left(\omega^{\alpha} a, \omega^{\beta} b\right) & =\pi\left(\phi \cdot \omega^{\omega^{\gamma} f_{k}} a, \lambda \eta b\right) \\
& =\pi\left(\pi\left(\phi, \omega^{\omega^{\gamma} k f_{k}}\right), \pi(\lambda, \eta)\right) a b \\
& =\pi\left(\pi(\phi, \lambda), \pi\left(\omega^{\omega^{\gamma} f_{k}}, \eta\right)\right) a b \\
& =\pi\left(\pi(\phi, \lambda), \omega^{{ }^{\gamma} k f_{k} \eta}\right) a b \\
& =\pi(\phi, \lambda) \omega^{\omega^{\gamma_{k f}} \eta} a b .
\end{aligned}
$$

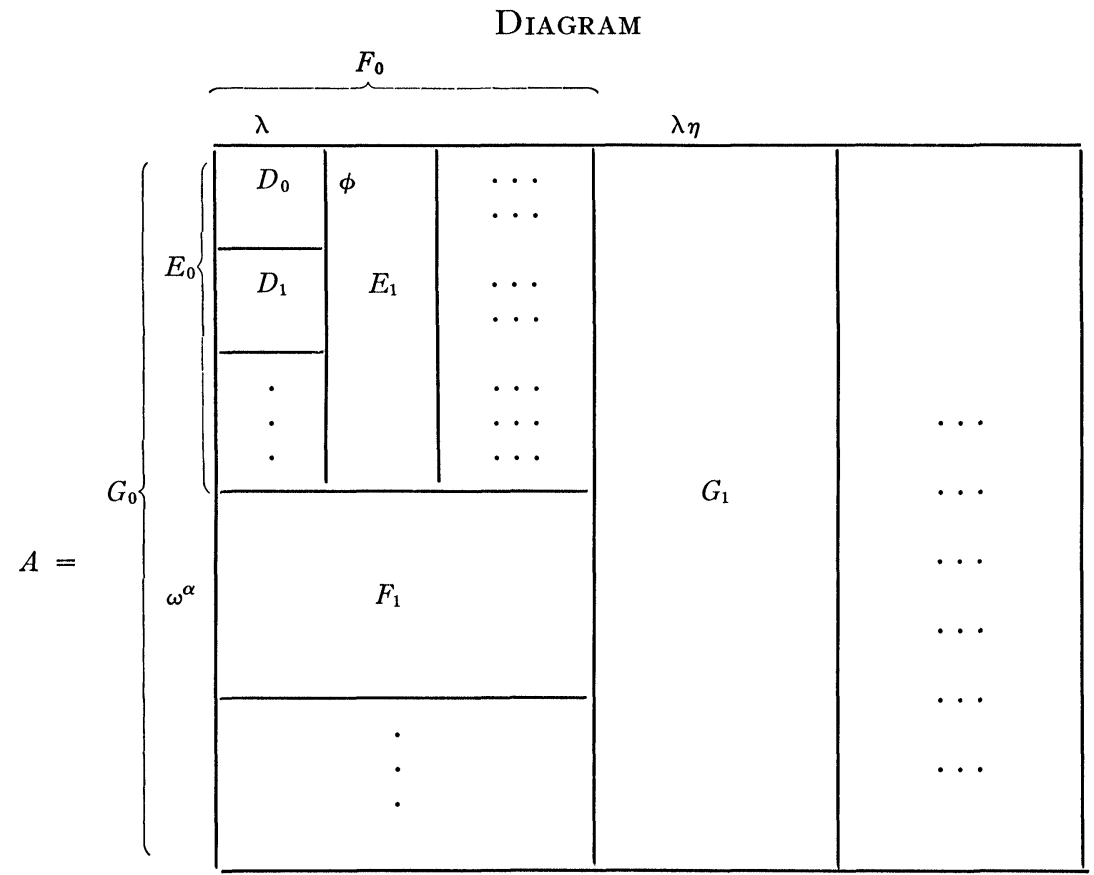

By our induction hypothesis, there exists an ordered $\phi, \lambda$-block $D_{0}$ of order type $\pi(\phi, \lambda)$. We may construct congruent ordered $\phi, \lambda$ blocks $D_{\alpha}, 0 \leqq \alpha<\omega^{{ }{ }^{\gamma} f_{k}}$, as in the Diagram, such that $D_{\alpha}<D_{\beta}$ for $\alpha<\beta$.

Let $E_{0}$ be the block of all the $D_{\alpha} . E_{0}$ is an ordered $\omega^{\alpha}, \lambda$-block. The

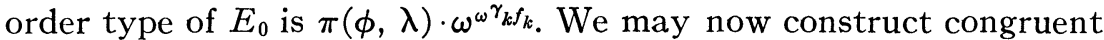
ordered $\omega^{\alpha}, \lambda$-blocks $E_{\beta}, 0 \leqq \beta<\eta$, as in the Diagram, such that $E_{\beta}<E_{\delta}$, for $\beta<\delta$. 
Let $F_{0}$ be the block of all the $E_{\beta}$. Then $F_{0}$ is an ordered $\omega^{\alpha}, \lambda \eta$-block. The order type of $F_{0}$ is

$$
\pi(\phi, \lambda) \cdot \omega^{\omega \gamma_{k} f_{k} \cdot \eta}
$$

Now we construct congruent ordered $\omega^{\alpha}, \lambda \eta$-blocks $F_{i}, 0 \leqq i<a$, such that $F_{i}<F_{j}$, for $i<j$.

Let $G_{0}$ be the block of all the $F_{i}$. Then $G_{0}$ is an ordered $\omega^{\alpha} a, \lambda_{\eta}$ block. The order type of $G_{0}$ is $\pi(\phi, \lambda) \omega^{\omega^{\gamma} f_{k}} \cdot \eta a$. We construct congruent ordered $\omega^{\alpha} a, \lambda \eta$-blocks $G_{i}, 0 \leqq i<b$, as in the Diagram, such that $G_{i}<G_{j}$, for $i<j$.

Let $A$ be the block of all the $G_{i}$. $A$ is an ordered $\omega^{\alpha} a, \omega^{\beta} b$-block. The order type of $A$ is

$$
\pi(\phi, \lambda) \omega^{\omega^{\gamma} k f_{k} \cdot \eta \cdot a b}=\pi\left(\omega^{\alpha} a, \omega^{\beta} b\right)=\pi(\tau, \mu) .
$$

III is an immediate consequence of I and II, and IV is an immediate consequence of $\mathrm{I}$.

3. Well-ordered subsets of ordered Abelian groups. We prove the following theorem.

Theorem 3. Let $G$ be an ordered Abelian group. Let $T$ be a wellordered (according to size) set of non-negative elements in $G$, containing 0 , of order type $\beta=\tau+n$, where $\tau$ is a limit ordinal or 0 , and $n$ is an integer greater than or equal to 0 . Let $M$ be the set of all finite sums of elements in T. Then:

I. $M$ is well-ordered.

II. $M$ has order type less than or equal to $\pi\left(\omega^{\beta}, \tau^{\tau}\right){ }^{7}$

\section{Proof of I.}

Case 1. There exists an integer $N$ such that each element in $M$ is the sum of $N$ elements in $T$.

Let $M_{i}$ be the set of sums of at most $i$ elements in $T$. Then $M_{i}$ is well-ordered if $i=1$. Assume that $M_{k-1}$ is well-ordered. Suppose that $M_{k}$ is not well-ordered. Then there exists a chain of elements in $M_{k}$, $a_{1}>a_{2}>\cdots$, of order type $\omega^{*}$. Let $a_{i}=c_{i}+d_{i}$, where $c_{i}$ is in $M_{k-1}$, and $d_{i}$ is in $M_{1}=T$. Then $c_{1}+d_{1}>c_{2}+d_{2}>\ldots$. The $c_{i}$ are wellordered. Likewise the $d_{i}$ are well-ordered. Hence this chain can not exist, that is, $M_{k}$ is well-ordered.

Case 2. There exists no integer $N$ such that each element in $M$ is the sum of $N$ elements in $T$.

Let $T$ be the set $\left\{t_{\alpha}\right\}$, where $t_{\alpha}<t_{\beta}$ for $\alpha<\beta$. Let $D_{\alpha}$ be the subgroup of $G$ that is generated by $t_{\alpha}$ and all $g$ in $G$ such that $0 \leqq g<t_{\alpha}$. Then

\footnotetext{
${ }^{7}$ If $\beta$ is an integer, then a better bound of the order type of $M$ is $\pi\left(\omega^{\beta-1}, \tau^{\tau}\right)=\omega^{\beta-1}$.
} 
$0=D_{0} \leqq D_{1} \leqq D_{2} \leqq \ldots$. We pick out the well-ordered set $R$ of all the different $D_{\alpha} . R=\left\{R_{0}, R_{1}, \cdots\right\} ; 0=R_{0}<R_{1}<\cdots \leqq G$.

Let $M(\alpha)$ be the set of all elements in $M$ such that each may be expressed as a finite sum of elements in $T$, all of which are in $R_{\alpha}$.

$M(0)$ consists of the single element 0 and so is well-ordered. Assume that $M(\alpha)$ is well-ordered for all $\alpha<\gamma$. Suppose that $M(\gamma)$ is not wellordered. Then there exists a chain in $M(\gamma), a_{1}>a_{2}>\cdots$, of order type $\omega^{*}$.

(a) Only a finite number of the $a_{i}$ are in $M(\gamma)$ but not in an $M(\alpha)$, $\alpha<\gamma$.

In this case, we may throw out this finite number of $a_{i}$ and then we have a chain, $c_{1}>c_{2}>\cdots$, of order type $\omega^{*}$, where each $c_{i}$ is in an $M(\alpha), \alpha<\gamma$. Let $c_{1}$ be in $M(\rho), \rho<\gamma$. By our induction hypothesis, there exists a $c_{i}$, say $c_{k}$, not in $M(\rho)$. But then $c_{k}>c_{1}$, a contradiction.

(b) An infinite number of the $a_{i}$ are in $M(\gamma)$ but not in any $M(\alpha)$, $\alpha<\gamma$.

In this case, we may choose a chain, $c_{1}>c_{2}>\cdots$, of order type $\omega^{*}$, where each $c_{i}$ is in $M(\gamma)$ but not in any $M(\alpha), \alpha<\gamma$.

Let $c_{i}=f_{i}+g_{i}$, where $f_{i}$ is in an $M(\alpha), \alpha<\gamma$, and $g_{i}$ is a sum of terms in $T$, each of which is in $R_{\gamma}$ but not in an $R_{\alpha}, \alpha<\gamma$. We saw in (a) that the $f_{i}$ must be well-ordered. Hence the $g_{i}$ must not be wellordered, that is, we can choose a chain from the $g_{i}: g_{\alpha_{1}}>g_{\alpha_{2}}>\ldots$, of order type $\omega^{*}$. Each $g_{\alpha_{i}}$ is a sum of terms in $T$, all of which are in $R_{\gamma}$ but not in any $R_{\alpha}, \alpha<\gamma$.

Let $t$ be the smallest positive element in $T$ that is in $R_{\gamma}$ but not in an $R_{\alpha}, \alpha<\gamma$. There exists an integer $n$ such that $n t$ is greater than $g_{\alpha_{1}}$. According to Case 1 , there must exist a $g_{\alpha_{i}}$, say $g_{\alpha_{j}}$, that is the sum of more than $n$ positive elements in $T$. But then $g_{\alpha_{j}}>n t>g_{\alpha_{1}}$, a contradiction.

Before we proceed, we shall prove this lemma.

Lemma 1. Let $G$ be an ordered Abelian group. Let $T$ be a subset of non-negative elements in $G$, containing 0 , that is well-ordered (according to size) of order type $\beta$. Let $H_{k}$ be the set $\left\{\sum_{i=1}^{r} a_{i} n_{i}\right\}$, where the $n_{i}$ are non-negative integers such that $\sum_{i=1}^{r} n_{i} \leqq k$, the $a_{i}$ are in $T$, and $k$ is any positive integer. Then $H_{k}$ is well-ordered and has order type less than or equal to $\phi_{k}(\beta)=\pi_{k-1}\left(\pi_{k-2}\left(\cdots\left(\pi_{1}(\beta, \beta), \beta\right), \cdots\right), \beta\right)$, where the subscripts merely denote the number of $\pi$ 's.

Proof. $H_{k}$ is well-ordered, by Case 1 of Theorem 3. The order type of $H_{1}$ is $\beta$. Assume $H_{h-1}$ has order type less than or equal to $\phi_{h-1}(\beta)$. Let the elements in $H_{h-1}$ be $b_{0}, b_{1}, \cdots$, where $b_{\alpha}<b_{\delta}$ for $\alpha<\delta$. 
The set $R=\left\{b_{\alpha}+a_{\delta}\right\}$, all $b_{\alpha}$ in $H_{h-1}$, and all elements $a_{\delta}$ in $T$, certainly contains $H_{h}$. Theorem 2 implies that the order type of $H_{h}$ is less than or equal to $\pi\left(\phi_{h-1}(\beta), \beta\right)=\phi_{h}(\beta)$.

We now continue with the proof of Theorem 3 .

Proof OF II. Let $M^{\alpha}$ be the set of all elements in $M$ that are sums of elements $t_{\gamma}, 0 \leqq \gamma<\alpha$, in $T$. Note that $M^{\beta}=M$. Let $\alpha=\tau_{\alpha}+n_{\alpha}$, where $\tau_{\alpha}$ is a limit ordinal or 0 , and $n_{\alpha}$ is an integer greater than or equal to 0 .

$M^{1}$ has order type 1 . Assume that $M^{\alpha}, \alpha<\delta$, has order type less than or equal to $\pi\left(\omega^{\alpha}, \tau_{\alpha}^{\tau_{\alpha}}\right)$.

Case 1. $n_{\delta}>0$.

$M^{\delta-1}$ has order type less than or equal to $\pi\left(\omega^{\delta-1}, \tau_{\delta}^{\tau \delta}\right) . M^{\delta}$ is certainly contained in the set $\left\{a_{\alpha}+n t_{\delta-1}\right\}$, for all elements $a_{\alpha}$ in $M^{\delta-1}$, and $0 \leqq n<\infty$. Theorem 2 implies that the order type of $M^{\delta}$ is less than or equal to $\pi\left(\pi\left(\omega^{\delta-1}, \tau_{\delta}^{\tau \delta}\right), \omega\right)=\pi\left(\omega^{\delta}, \tau_{\delta}^{\tau \delta}\right)$.

Case 2. $n_{\delta}=0$, that is, $\tau_{\delta}=\delta$.

Suppose that $M^{\delta}$ has order type greater than $\pi\left(\omega^{\delta}, \delta^{\delta}\right)$. Then $M^{\delta}=S+R$, where $S$ is the segment of $M^{\delta}$ of order type $\pi\left(\omega^{\delta}, \delta^{\delta}\right)$.

Let $b=t_{\alpha_{1}} m_{1}+\cdots+t_{\alpha_{s}} m_{s}, \alpha_{1}<\alpha_{2}<\cdots<\alpha_{s}<\delta$, be the smallest element in $R$, where the $m_{i}$ are positive integers. Let $\alpha_{s}=\mu+m$, where $\mu$ is a limit ordinal or zero and $m$ is an integer greater than or equal to 0 . Let $n$ be an integer such that $n t_{\alpha_{s}}$ is greater than $b$.

Let $H_{n}$ be the set of all elements $\sum_{i=1}^{r} t_{\beta_{i}} n_{i}$ in $M^{\delta}$, such that $r \leqq n$, $\sum_{i=1}^{r} n_{i} \leqq n$, and each $\beta_{i}<\delta$. By Lemma 1 , it is seen that the order type of $H_{n}$ is less than or equal to $\phi_{n}(\delta)=\pi_{n-1}\left(\pi_{n-2}\left(\cdots\left(\pi_{1}(\delta, \delta), \delta\right)\right.\right.$ $\cdots), \delta)$.

Let $\delta=\mathrm{w}^{\delta_{1}} e_{1}+\cdots+\omega^{\delta_{q}} e_{q}$ and $\delta_{1}=\omega^{\rho_{1} v_{1}}+\cdots+\omega^{\rho_{z}} \mathrm{v}_{z}$. Then $\delta<\omega^{\omega} \rho_{1\left(v_{1}+1\right)}$ and $\phi_{n}(\delta)<\omega^{\omega \rho^{\rho}\left(v_{1}+1\right) n}$.

Let $x$ be any element in $S$. Then $x=t_{\lambda_{1}} r_{1}+\cdots+t_{\lambda_{v}} r_{v}+t_{\lambda_{\nu+1}+1} r_{v+1}$ $+\cdots+t_{\lambda_{e}} r_{e}$ where $\lambda_{1}<\cdots<\lambda_{v}<\alpha_{s} \leqq \lambda_{v+1}<\cdots<\lambda_{e}<\delta$. Let $f=t_{\lambda_{1}} r_{1}$ $+\cdots+t_{\lambda_{v}} r_{v}$, and $g=t_{\lambda_{v+1}} r_{v+1}+\cdots+t_{\lambda_{e}} r_{e}$. Then $f$ is in $M^{\alpha_{s}}$. Since $x$ is less than $b$, it follows that $g$ is less than $b$. Hence $r_{v+1}+\cdots+r_{e} \leqq n$. And so $g$ is in $H_{n}$. Therefore the set $S$ is contained in the set $\left\{b_{\alpha}+c_{\rho}\right\}$, for all elements $b_{\alpha}$ in $H_{n}$, and for all elements $c_{\rho}$ in $M^{\alpha_{s}}$. Theorem 2 and our induction hypothesis imply that $S$ has order type less than or equal to $\pi\left(\pi\left(\omega^{\alpha_{s}}, \mu^{\mu}\right), \omega^{\omega \rho_{1}\left(v_{1}+1\right) n}\right) \leqq \pi\left(\pi\left(\omega^{\delta}, \delta^{\mu}\right), \delta^{v_{1} n+n}\right)$.

(1) $\pi\left(\pi\left(\omega^{\delta}, \delta^{\mu}\right), \delta^{v_{1} n+n}\right)=\pi\left(\omega^{\delta}, \pi\left(\delta^{\mu}, \delta^{j}\right)\right)$, where $j=v_{1} n+n$ is an integer, $\pi\left(\delta^{\mu}, \delta^{j}\right) \leqq \pi\left(\left(\omega^{\delta_{1}}\left(e_{1}+1\right)\right)^{\mu},\left(\omega^{\delta_{1}}\left(e_{1}+1\right)\right)^{j}\right) . \delta_{1}>0$, since $\delta$ is a limit ordinal. Hence $\left(\omega^{\delta_{1}}\left(e_{1}+1\right)\right)^{\mu}=\omega^{\delta_{1} \mu}$, since $\mu$ is a limit ordinal. $\left(\omega^{\delta_{1}}\left(e_{1}+1\right)\right)^{j}$ $=\omega^{\delta_{1 j} j}\left(e_{1}+1\right)$,

$$
\pi\left(\delta^{\mu}, \delta^{j}\right) \leqq \pi\left(\omega^{\delta_{1 \mu}}, \omega^{\delta_{1 j}}\left(e_{1}+1\right)\right)=\omega^{\sigma\left(\delta_{1 \mu}, \delta_{1} j\right)}\left(e_{1}+1\right) .
$$




$$
\begin{array}{r}
\delta_{1} \omega=\omega^{\rho_{1}+1}, \text { and } \delta_{1} j=\omega^{\rho_{1}} v_{1} j+\omega^{\rho 2 v_{2}}+\cdots+\omega^{\rho_{z} v_{z}}<\omega^{\rho_{1}}\left(v_{1} j+1\right) \\
\sigma\left(\delta_{1} \mu, \delta_{1} j\right)<\sigma\left(\delta_{1} \mu, \omega^{\rho_{1}}\left(v_{1} j+1\right)\right)<\delta_{1} \mu+\omega^{\rho_{1}+1}=\delta_{1} \mu+\delta_{1} \omega .
\end{array}
$$

By $(2), \pi\left(\delta^{\mu}, \delta^{j}\right)<\omega^{\delta_{1} \mu+\delta_{1} \omega}=\left(\omega^{\delta_{1}}\right)^{(\mu+\omega)} \leqq \delta^{\mu+\omega} \leqq \delta^{\delta}$.

Hence by (1), the order type of $S$ is less than $\pi\left(\omega^{\delta}, \delta^{\delta}\right)$. This is a contradiction since $S$ was the segment of $M^{\delta}$ of order type $\pi\left(\omega^{\delta}, \delta^{\delta}\right)$.

UNIVERSITY OF ILLINOIS

\section{A CHARACTERIZATION OF ABSOLUTE NEIGHBORHOOD RETRACTS}

\section{RALPH H. FOX}

By an absolute neighborhood retract (ANR) I mean a separable metrizable space which is a neighborhood retract of every separable metrizable space which contains it and in which it is closed. This generalization of Borsuk's original definition ${ }^{1}$ was given by Kuratow$\mathrm{ski}^{2}$ for the purpose of enlarging the class of absolute neighborhood retracts to include certain spaces which are not compact. The space originally designated by Borsuk as absolute neighborhood retracts (or $\Re$-sets) will now be referred to as compact absolute neighborhood retracts. Many of the properties of compact ANR-sets hold equally for the more general ANR-sets. ${ }^{3}$

The Hilbert parallelotope $Q$, that is, the product of the closed unit interval $[0,1]$ with itself a countable number of times is a "universal" compact ANR in the sense that every compact ANR is homeomorphic to a neighborhood retract of $Q$. The classical theory of Borsuk makes good use of the imbedding of compact ANR-sets in $Q$. The problem solved here is that of finding a "universal" ANR.

Received by the editors June 28, 1941.

${ }^{1}$ Fundamenta Mathematicae, vol. 19 (1932), pp. 220-242.

${ }^{2}$ Fundamenta Mathematicae, vol. 24 (1935), p. 270, Footnote 1.

3 Ibid., pp. 272, 276, and 277, and Footnote 1, p. 279 and Footnote 3. Note that Theorem 12, Fundamenta Mathematicae, vol. 19 (1932), p. 229, is not true for general ANR-sets. In fact let $A=\sum S_{n}$ where $S_{n}$ is the plane circle of radius $2^{-n}$ and center $\left(3 \cdot 2^{-n}, 0\right)$; let $f(x, y)=(x,|y|)$ for $(x, y) \in A$ and let

$$
f_{n}(x, y)=\left\{\begin{array}{l}
(x,|y|), \text { for }(x, y) \in A-S_{n}, \\
(x, y), \quad \text { for }(x, y) \in S_{n} .
\end{array}\right.
$$

Then $f_{n} \rightarrow f$ in $A^{A} ; f$ can be extended to the half-plane $\{x>0\}$, but none of the maps $f_{n}$ can. $A$ is an ANR-set. Theorem 16, Fundamenta Mathematicae, vol. 19 (1932), p. 230, is also false for general ANR-sets.

${ }^{4}$ Fundamenta Mathematicae, vol. 19 (1932), p. 223. 